PROCEEDINGS OF THE

AMERICAN MATHEMATICAL SOCIETY

Volume 128, Number 2, Pages 583-588

S 0002-9939(99)05039-X

Article electronically published on July 6, 1999

\title{
ON COMPACT SUBSETS IN COECHELON SPACES OF INFINITE ORDER
}

\author{
ANGELA A. ALBANESE
}

(Communicated by Dale Alspach)

\begin{abstract}
For coechelon spaces $k_{\infty}(v)$ of infinite order it is proved that every compact subset of $k_{\infty}(v)$ is contained in a closed absolutely convex hull of some null sequence if and only if the matrix $v$ is regularly decreasing.
\end{abstract}

In connection with the study of some interesting problems on Montel maps which are closely connected to the classical Grothendieck question on completeness of regular LB-spaces [PB, Problem 13.8.6] and the problem of bornologicity of $C(K, E)$ with $E$ an LB-space [S, Chapter IV], Dierolf and Domański [DD2, Example 3.1] gave an example of a coechelon LB-Montel space of infinite order which has compact sets not contained in closed absolutely convex hulls of any null sequence. Consequently, the well-known characterization of compact sets in Fréchet spaces [J, Theorem 9.4.2] turns out to be not generally true in the LB setting.

The purpose of this note is to show that for coechelon spaces $k_{\infty}(v)$ of order $\infty$ the condition $v$ regularly decreasing [BMS, Definition 3.1] is necessary and sufficient for every compact set to be contained in a closed absolutely convex hull of some null sequence.

For more information on Montel maps and related questions the reader is referred to [DD1], [DD2], [DD3] and [D].

In what follows we recall some notation.

Let $E$ be a Fréchet space with a fundamental system of seminorms $\left(\|\|_{n}\right)_{n}$; then the inductive dual $E_{i}^{\prime}$ is defined to be $\operatorname{ind}_{n} E_{n}^{\prime}$, where the $E_{n}$ are the completions of the normed spaces $\left(E /\right.$ ker \|\|$\left._{n},\|\|_{n}\right)$. It is known that algebraically $E_{i}^{\prime}=E^{\prime}$, the inclusion map $E_{i}^{\prime} \hookrightarrow E_{\beta}^{\prime}$ is continuous and $E_{i}^{\prime}$ is the bornological space associated with $E_{\beta}^{\prime}$, i.e., $E_{i}^{\prime}=\left(E^{\prime}, \beta\left(E^{\prime}, E^{\prime \prime}\right)\right)\left[\mathrm{J}\right.$, Theorem 13.4.2] $\left(E^{\prime}\right.$ and $E_{\beta}^{\prime}$ denote the topological dual and the strong dual of $E$, resp.).

We also recall that an LB-space $E=\operatorname{ind}_{n} E_{n}$ is called boundedly retractive (respectively, compactly regular) if, and only if, for each bounded (respectively, compact) subset $B$ of $E$ there is $n \in \mathbf{N}$ such that $B \subset E_{n}$ and $E_{n}$ and $E$ induce the same topology on $B$ (see [PB, Definitions 8.5.32-(ii), -(iii)]). It is clear that a boundedly retractive LB-space is compactly regular. On the other hand, in $[\mathrm{N}]$ it is proved that these conditions are also equivalent.

Received by the editors August 12, 1997 and, in revised form, April 14, 1998.

1991 Mathematics Subject Classification. Primary 46A45; Secondary 46A50.

Key words and phrases. Compact set, coechelon space of infinite order, regularly decreasing. Research partially supported by the Italian MURST. 
Let $a=\left(a_{n}(i)\right)_{n}$ and $v=\left(v_{n}(i)\right)_{n}$ be matrices on an index set $I$, with $v_{n}(i) \geq$ $v_{n+1}(i)>0$ and $a_{n}(i)=\frac{1}{v_{n}(i)}$ for all $i \in I$ and $n \in \mathbf{N}$. The Köthe echelon space of order 1 (associated to $a$ ) and the Köthe coechelon space of order $\infty$ (associated to $v$ ) are defined by

$$
\lambda_{1}(a):=\left\{\left(x_{i}\right)_{i \in I}:\left\|\left(x_{i}\right)_{i}\right\|_{n}^{1}:=\sum_{i \in I} a_{n}(i)\left|x_{i}\right|<+\infty \text { for all } n \in \mathbf{N}\right\}
$$

and

$$
k_{\infty}(v):=\left\{\left(x_{i}\right)_{i \in I}:\left\|\left(x_{i}\right)_{i}\right\|_{n}^{\infty}:=\sup _{i \in I} v_{n}(i)\left|x_{i}\right|<+\infty \text { for some } n \in \mathbf{N}\right\},
$$

respectively. Clearly, $k_{\infty}(v)=\operatorname{ind}_{n} l_{\infty}\left(v_{n}\right)\left(l_{\infty}(w):=\left\{\left(x_{i}\right)_{i \in I}: \sup _{i \in I} w(i)\left|x_{i}\right|<\right.\right.$ $+\infty\}$ for any positive function $w$ on $I$ ); also, $k_{\infty}(v)=\left(\lambda_{1}(a)\right)_{i}^{\prime}$ (see [BMS, Corollary $2.8])$ and hence it is a regular and complete LB-space.

If $\bar{V}$ is the maximal Nachbin family of positive functions on $I$ associated to $v$ which is defined by

$$
\bar{V}:=\left\{\bar{v}: I \rightarrow\left[0,+\infty\left[: \sup _{i \in I} \frac{\bar{v}(i)}{v_{n}(i)}<+\infty \text { for all } n \in \mathbf{N}\right\},\right.\right.
$$

then, by [BMS, Theorem 2.7], $\left(\lambda_{1}(a)\right)_{\beta}^{\prime}$ coincides algebraically and topologically with

$$
K_{\infty}(\bar{V}):=\operatorname{proj}_{\bar{v} \in \bar{V}} l_{\infty}(\bar{v}) .
$$

So, algebraically $k_{\infty}(v)=K_{\infty}(\bar{V})$, the inclusion map $k_{\infty}(v) \hookrightarrow K_{\infty}(\bar{V})$ is continuous and they have the same bounded sets. In particular, since every compact set in $K_{\infty}(\bar{V})=\left(\lambda_{1}(a)\right)_{\beta}^{\prime}$ is separable and metrizable by [CO, Corollary 1.2], the topology of $K_{\infty}(\bar{V})$ coincides with the one of $k_{\infty}(v)$ on these sets (cf. [PB, Proposition 8.3.12]); hence, every compact set in $K_{\infty}(\bar{V})$ is also a compact set in $k_{\infty}(v)$.

Finally, we recall that the matrix $v$ is called regularly decreasing [BMS, Definition $3.1]$ if, and only if, given $n \in \mathbf{N}$ there is $m \geq n$ such that

$$
\forall I_{0} \subset I \text { with } \inf _{i \in I_{0}} \frac{v_{m}(i)}{v_{n}(i)}>0 \text {, we have also } \inf _{i \in I_{0}} \frac{v_{k}(i)}{v_{n}(i)}>0 \text { for all } k \geq m \text {. }
$$

By [BMS, Theorem 3.4] $v$ is regularly decreasing if, and only if, $\lambda_{1}(a)$ is quasinormable if, and only if, $k_{\infty}(v)$ is a boundedly retractive LB-space and hence if, and only if, it is a compactly regular LB-space.

For all undefined notation we refer to $[\mathrm{PB}]$ and $[\mathrm{BMS}]$.

We are now able to state and prove our result.

Theorem. Let $k_{\infty}(v)$ be a coechelon space of order $\infty$. Then the following conditions are equivalent:

(i) $v$ is regularly decreasing;

(ii) every compact set in $k_{\infty}(v)$ is contained in a closed absolutely convex hull of some null sequence.

Proof. We establish that (i) implies (ii). Let $K$ be a compact set in $k_{\infty}(v)$. Since $v$ is regularly decreasing, $k_{\infty}(v)$ is boundedly retractive. Thus $K$ is contained in $l_{\infty}\left(v_{n}\right)$ for some $n \in \mathbf{N}$ and $l_{\infty}\left(v_{n}\right)$ and $k_{\infty}(v)$ induce the same topology on $K$; hence, it is a compact subset of $l_{\infty}\left(v_{n}\right)$. Since $l_{\infty}\left(v_{n}\right)$ is a Banach space, by [J, Theorem 9.4.2] 
$K$ is contained in a closed absolutely convex hull of some null sequence in $l_{\infty}\left(v_{n}\right)$ and the result follows.

Next, we show that (ii) implies (i). Suppose that $v$ is not regularly decreasing. Then:

$$
\begin{gathered}
\exists n_{o} \in \mathbf{N} \forall m>n_{0} \exists J \subset I \text { and } \exists k>m \text { such that } \\
\inf _{i \in J} \frac{v_{m}(i)}{v_{n_{0}}(i)}>0 \text { and } \inf _{i \in J} \frac{v_{k}(i)}{v_{n_{0}}(i)}=0 .
\end{gathered}
$$

Proceeding by induction as in [V, p. 232], we can find a sectional subspace of $k_{\infty}(v)$ which is isomorphic to $k_{\infty}(\tilde{v})$, with $\tilde{v}=\left(\tilde{v}_{n}(i, j)\right)_{n}$ a matrix on $\mathbf{N} \times \mathbf{N}$ satisfying:

$$
\begin{gathered}
\tilde{v}_{1}(i, j)=1, \forall i, j \in \mathbf{N} ; \\
\lim _{j \rightarrow \infty} \tilde{v}_{n+1}(n, j)=0, \forall n \in \mathbf{N} ; \\
\forall n \in \mathbf{N} \forall i \geq n \exists \alpha_{n, i}>0 \inf _{j \in \mathbf{N}} \tilde{v}_{n}(i, j)>\alpha_{n, i} .
\end{gathered}
$$

Since $k_{\infty}(\tilde{v})$ is complemented in $k_{\infty}(v)$ as it is easy to see, we can restrict our attention to $k_{\infty}(\tilde{v})\left(k_{\infty}(v)\right.$ satisfies (ii) $\Rightarrow$ every complemented subspace of $k_{\infty}(v)$ satisfies (ii)). For technical reasons we modify the weights by defining $v_{n}^{\star}(i, j):=i^{-2} \tilde{v}_{n}(i, j)$. Clearly, $k_{\infty}(\tilde{v})$ is isomorphic to $k_{\infty}\left(v^{\star}\right)$, with $v^{\star}=\left(v_{n}^{\star}(i, j)\right)_{n}$. Moreover, $v^{\star}$ satisfies the following conditions:

$$
\begin{gathered}
v_{1}^{\star}(i, j)=i^{-2}, \forall i, j \in \mathbf{N} \\
\lim _{j \rightarrow \infty} v_{n+1}^{\star}(n, j)=0, \forall n \in \mathbf{N} ; \\
\forall n \in \mathbf{N} \forall i \geq n \exists \alpha_{n, i}>0 \inf _{j} v_{n}^{\star}(i, j)>i^{-2} \alpha_{n, i} .
\end{gathered}
$$

We will show that the set $C:=\left\{\left(x_{i j}\right)_{i, j \in \mathbf{N}}: \sup _{i, j \in \mathbf{N}}\left|x_{i j}\right| i^{-1} \leq 1\right\}$ is a compact set in $k_{\infty}\left(v^{\star}\right)$ which is not contained in the closed absolutely convex hull of any null sequence (clearly, $C \subset B_{1}$, where $B_{1}$ denotes the closed unit ball of $l_{\infty}\left(v_{1}^{\star}\right)$ ).

Let $\bar{V}$ be the maximal Nachbin family of positive functions on $\mathbf{N} \times \mathbf{N}$ associated to $v^{\star}$. Since every compact set in $K_{\infty}(\bar{V})$ is also a compact set in $k_{\infty}\left(v^{\star}\right)$, we have only to show that $C$ is compact in $K_{\infty}(\bar{V})$, i.e., $C$ is compact in $l_{\infty}(\bar{v})$ for every $\bar{v} \in \bar{V}$

Now, let $\bar{v} \in \bar{V}$. By (a) and (b), it follows that:

$$
\begin{gathered}
\lim _{i \rightarrow \infty} i \sup _{j \in \mathbf{N}} \bar{v}(i, j)=0 \text { and } i \bar{v}(i, j) \leq c<+\infty, \forall i, j \in \mathbf{N} ; \\
\lim _{j \rightarrow \infty} n \bar{v}(n, j)=0, \forall n \in \mathbf{N} .
\end{gathered}
$$

Next, let $\left(x^{r}\right)_{r}$ be a sequence of $C$. Then, for each $i$ and $j \in \mathbf{N}, \sup _{r} i^{-1}\left|x_{i j}^{r}\right| \leq 1$. This implies that (first by passing to subsequences and then by diagonalization) there are a sequence $x=\left(x_{i j}\right)_{i, j \in \mathbf{N}}$ in $\mathbf{R}$ and a subsequence $\left(x^{r^{\prime}}\right)_{r^{\prime}}$ of $\left(x^{r}\right)_{r}$ so that, for each $i$ and $j \in \mathbf{N}$,

$$
\lim _{r^{\prime} \rightarrow \infty} i^{-1}\left|x_{i j}^{r^{\prime}}-x_{i j}\right|=0
$$


hence, for each $i$ and $j \in \mathbf{N}$,

$$
i^{-1}\left|x_{i j}\right| \leq 1 \quad \text { and } \quad i^{-1}\left|x_{i j}^{r^{\prime}}-x_{i j}\right| \leq 2
$$

Clearly, $x \in C$. We check that $\left(x^{r^{\prime}}\right)_{r^{\prime}}$ converges to $x$ in $l_{\infty}(\bar{v})$. Given any $\varepsilon>0$, by (4), there is $i_{0} \in \mathbf{N}$ such that

$$
\sup _{j \in \mathbf{N}} i \bar{v}(i, j)<\varepsilon / 6 \quad \text { for all } i \geq i_{0} .
$$

It follows, by (7), that

$$
\begin{aligned}
\sup _{i, j \in \mathbf{N}}\left|x_{i j}^{r^{\prime}}-x_{i j}\right| \bar{v}(i, j) & \leq \sup _{i<i_{0}} \sup _{j \in \mathbf{N}}\left|x_{i j}^{r^{\prime}}-x_{i j}\right| \bar{v}(i, j)+\sup _{i \geq i_{0}} \sup _{j \in \mathbf{N}} i^{-1}\left|x_{i j}^{r^{\prime}}-x_{i j}\right| i \bar{v}(i, j) \\
& \leq \sup _{i<i_{0}} \sup _{j \in \mathbf{N}}\left|x_{i j}^{r^{\prime}}-x_{i j}\right| \bar{v}(i, j)+2 \sup _{i \geq i_{0}} \sup _{j \in \mathbf{N}} i \bar{v}(i, j) \\
& \leq \sup _{i<i_{0}} \sup _{j \in \mathbf{N}}\left|x_{i j}^{r^{\prime}}-x_{i j}\right| \bar{v}(i, j)+\varepsilon / 3 .
\end{aligned}
$$

for every $r^{\prime} \in \mathbf{N}$. On the other hand, by $(5), \lim _{j \rightarrow \infty} i \bar{v}(i, j)=0$ for $i=1, \ldots, i_{0}-1$ and hence there is $j_{0} \in \mathbf{N}$ such that $i \bar{v}(i, j)<\varepsilon / 6$ for each $i=1, \ldots, i_{0}-1$ and $j \geq j_{0}$. This implies by (4), (7) and (8) that, for each $r^{\prime} \in \mathbf{N}$,

$$
\begin{aligned}
\sup _{i, j \in \mathbf{N}}\left|x_{i j}^{r^{\prime}}-x_{i j}\right| \bar{v}(i, j) \leq & \sup _{i<i_{0}} \sup _{j<j_{0}} i^{-1}\left|x_{i j}^{r^{\prime}}-x_{i j}\right| i \bar{v}(i, j) \\
& +\sup _{i<i_{0}} \sup _{j \geq j_{0}} i^{-1}\left|x_{i j}^{r^{\prime}}-x_{i j}\right| i \bar{v}(i, j)+\varepsilon / 3 \\
\leq & c \sup _{i<i_{0}} \sup _{j<j_{0}} i^{-1}\left|x_{i j}^{r^{\prime}}-x_{i j}\right|+2 \sup _{i<i_{0}} \sup _{j \geq j_{0}} i \bar{v}(i, j)+\varepsilon / 3 \\
\leq & c \sup _{i<i_{0}} \sup _{j<j_{0}} i^{-1}\left|x_{i j}^{r^{\prime}}-x_{i j}\right|+2 \varepsilon / 3,
\end{aligned}
$$

where by (6) $\lim _{r^{\prime} \rightarrow \infty} \sup _{i<i_{0}} \sup _{j<j_{0}} i^{-1}\left|x_{i j}^{r^{\prime}}-x_{i j}\right|=0$ and hence there is $r_{0}^{\prime} \in \mathbf{N}$ such that, for each $r^{\prime} \geq r_{0}^{\prime}$,

$$
\sup _{i<i_{0}} \sup _{j<j_{0}} i^{-1}\left|x_{i j}^{r^{\prime}}-x_{i j}\right|<\varepsilon / 3 c .
$$

Thus, by (9), we get that

$$
\sup _{i, j \in \mathbf{N}}\left|x_{i j}^{r^{\prime}}-x_{i j}\right| \bar{v}(i, j)<\varepsilon
$$

for all $r^{\prime} \geq r_{0}^{\prime}$.

Since $\varepsilon$ is arbitrary, this means that $\left(x^{r^{\prime}}\right)_{r^{\prime}}$ converges to $x$ in $l_{\infty}(\bar{v})$, where $x \in C$. We can therefore conclude that $C$ is a compact subset of $l_{\infty}(\bar{v})$. Since $\bar{v}$ is also arbitrary, $C$ is a compact subset of $K_{\infty}(\bar{V})$ too.

It remains to show that $C$ is not contained in the closed absolutely convex hull of any null sequence in $k_{\infty}\left(v^{\star}\right)$. For this we proceed in a similar way as in Example 3.1 [DD2], which is based on an idea of L. Frerick and J. Wengenroth. 
Assume that

$$
C \subset \overline{\operatorname{absconv}\left\{y_{k}: k \in \mathbf{N}\right\}}:=C_{1},
$$

where $\left(y_{k}\right)_{k} \in c_{0}\left(k_{\infty}\left(v^{\star}\right)\right)$. Since $k_{\infty}\left(v^{\star}\right)$ is a regular LB-space, $C_{1} \subseteq \rho B_{n}$ for some $n>1$ and $\rho>0\left(B_{n}\right.$ denotes the closed unit ball of $\left.l_{\infty}\left(v_{n}^{\star}\right)\right)$.

Let $P: k_{\infty}\left(v^{\star}\right) \rightarrow k_{\infty}\left(v^{\star}\right)$ be the projection defined by

$$
P\left(\left(x_{i j}\right)_{i, j}\right)=\left(z_{i j}\right)_{i, j}, \quad z_{i j}:= \begin{cases}0, & \text { if } i \neq n ; \\ x_{n j}, & \text { if } i=n .\end{cases}
$$

We then have $P(C) \subseteq P\left(B_{1}\right) \subseteq P\left(B_{n}\right)$. By (c), we have that $P\left(B_{n}\right) \subseteq n \alpha_{n, n}^{-1} P(C)$. Put $D:=P(C)$ and $E_{D}:=\operatorname{span} D$ (clearly, $\left(E_{D}, p_{D}\right)$ is a Banach space).

Next, we define a map

$$
T: l^{1} \rightarrow E_{D}, \quad T\left(\left(a_{k}\right)_{k}\right):=\sum_{k=1}^{\infty} a_{k} P y_{k} .
$$

Since $T\left(B_{l^{1}}\right) \subseteq \rho P\left(B_{n}\right) \subseteq \rho n \alpha_{n, n}^{-1} D, T$ is continuous. Also, since $\left(P y_{k}\right)_{k} \in$ $c_{0}\left(k_{\infty}\left(v^{\star}\right)\right)$, the restriction of $T$ to the closed unit ball of $l^{1}, B_{l^{1}}$, endowed with the weak topology $\sigma\left(l^{1}, c_{0}\right)$ is continuous (see [PB, Lemma 3.2.11]) and hence $T\left(B_{l^{1}}\right)$ is a compact absolutely convex subset of $k_{\infty}\left(v^{\star}\right)$. So, we have

$$
T\left(B_{l^{1}}\right) \supseteq \overline{\operatorname{absconv}\left\{P y_{k}: k \in \mathbf{N}\right\}} \supseteq D
$$

and hence $T$ is open. Now, $\left(E_{D}, p_{D}\right)$ is isomorphic to $l^{\infty}$ and $l^{1}$ is separable, thereby obtaining a contradiction.

Thus, (ii) implies (i) and this completes the proof.

As an immediate consequence, we obtain:

Remark. Every coechelon LB-Montel space $k_{\infty}(v)$ of order $\infty$ which is not a (DFS)space (and hence $\left(k_{\infty}(v)\right)_{\beta}^{\prime}=\lambda_{1}(a)$ is not quasinormable) has compact subsets not contained in closed absolutely convex hulls of any null sequence and hence so does the coechelon LB-Montel space constructed by Grothendieck and Köthe and considered in Example 3.1 of [DD2].

\section{REFERENCES}

[BMS] K. D. Bierstedt, R. Meise, W. Summers, Köthe sets and Köthe sequence spaces, pp. 27-91 in: "Functional Analysis, Holomorphy and Approximation Theory", North-Holland Math. Studies 71, Amsterdam 1982. MR 84f: 46011

[CO] B. Cascales and J. Orihuela, Metrizability of precompact subsets in (LF)-spaces, Proc. Roy. Soc. Edinburgh 103A (1986), 293-299. MR 88b:46003

[DD1] S. Dierolf and P. Domański, Factorization of Montel operators, Studia Math. 107 (1993), 15-32. MR 94i:46004

[DD2] S. Dierolf and P. Domański, Null Sequences in Coechelon Spaces, Math. Nachr. 184 (1997), 167-176. MR 98a:46013

[DD3] S. Dierolf and P. Domański, Bornological Spaces of Null Sequences, Arch. Math. 65 (1995), 46-52. MR 96d:46002

[D] P. Domański, On Spaces of Continuous Functions with Values in Coechelon Spaces, to appear in Rev. Real Acad. Sci. Exactas, Madrid.

[J] H. Jarchow, Locally convex spaces, Teubner Verlag, Stuttgart 1981. MR 83h:46008

$[\mathrm{N}] \quad \mathrm{H}$. Neus, Über die Regularitätsbegriffe induktiver lokalkonvexer Sequenzen, Manuscripta Math. 25 (1978), 135-145. MR 58:2125

[PB] P. Pérez Carreras and J. Bonet, Barrelled Locally Convex Spaces, North-Holland Math. Studies 131, Amsterdam 1987. MR 88j:46003 
[S] J. Schmets, Spaces of Vector-Valued Continuous Functions, Lect. Notes Math. 1003, Springer Verlag, Berlin 1983. MR 85g:46046

[V] M. Valdivia, Topics in Locally Convex Spaces, North-Holland Math. Studies 67, Amsterdam 1982. MR 84i: 46007

Dipartimento di Matematica "E. De Giorgi", Università di Lecce, C.P. 193, Via Per Arnesano, 73100, LeCce, Italy

E-mail address: albanese@ilenic.unile.it 